\title{
Solid Phase Epitaxial Re-Growth Of Sn Ion Implanted Germanium Thin Films
}

\author{
D. Giubertoni ${ }^{1)}$, E. Demenev ${ }^{1)}$, S. Gupta ${ }^{2)}$, Y. Jestin ${ }^{1)}$, F. Meirer ${ }^{1)}$, S. Gennaro ${ }^{1)}$, \\ E. Iacob $^{1)}$, G. Pepponi ${ }^{1)}$, G. Pucker ${ }^{1)}$, R. M. Gwilliam ${ }^{3)}$, C. Jeynes ${ }^{3)}$, J. L. Colaux ${ }^{3)}$, \\ K. C. Saraswat ${ }^{2)}$ and M. Bersani ${ }^{1)}$ \\ 1) CMM - Fondazione Bruno Kessler, Via Sommarive 18, 38123 Povo - Trento, Italy \\ 2) Department of Electrical Engineering, Stanford University, USA \\ ${ }^{3)}$ Ion Beam Centre, ATI, University of Surrey, Guildford, Surrey, GU27XH, UK
}

\begin{abstract}
Doping of Ge with Sn atoms by ion implantation and annealing by solid phase epitaxial re-growth process was investigated as a possible way to create $\mathrm{Ge}_{1-\mathrm{x}} \mathrm{Sn}_{\mathrm{x}}$ layers. Ion implantation was carried out at liquid nitrogen to avoid nano-void formation and three implant doses were tested: $5 \times 10^{15}, 1 \times 10^{15}$ and $5 \times 10^{14} \mathrm{at} / \mathrm{cm}^{2}$, respectively. Implant energy was set to $45 \mathrm{keV}$ and implants were carried out through an $11 \mathrm{~nm} \operatorname{SiN}_{\mathrm{x}} \mathrm{O}_{\mathrm{y}}$ film to prevent $\mathrm{Sn}$ out-diffusion upon annealing. This was only partially effective. Samples were then annealed in inert atmosphere either at $350^{\circ} \mathrm{C}$ varying anneal time or for $100 \mathrm{~s}$ varying temperature from 300 to $500^{\circ} \mathrm{C}$. SPER was effective to anneal damage without Sn diffusion at $350^{\circ}$ for samples implanted at medium and low fluences whereas the $5 \times 10^{15} \mathrm{at} / \mathrm{cm}^{2}$ samples remained with a $\sim 15 \mathrm{~nm}$ amorphous layer even when applying the highest thermal budget.
\end{abstract}

Keywords: germanium, $\mathrm{Ge}_{1-\mathrm{x}} \mathrm{Sn}_{\mathrm{x}}$, Tin, SPER.

PACS: 61.72.uf; 85.40.Ry; 61.72.S-.

\section{INTRODUCTION}

Semiconducting $\mathrm{Ge}_{1-\mathrm{x}} \mathrm{Sn}_{\mathrm{x}}$ alloy offers exciting avenues for band-gap and strain engineering in a silicon compatible technology because of its tunable bandgap [1] and possibility of large electron and hole mobilities [2]. Large hole mobilities in $\mathrm{Ge}_{1-\mathrm{x}} \mathrm{Sn}_{\mathrm{x}}$ based pMOSFETs have already been demonstrated [3, 4]. Due to the larger size of $\mathrm{Sn}$ atoms, use of $\mathrm{Ge}_{1-\mathrm{x}} \mathrm{Sn}_{\mathrm{x}}$ as source/drain in Ge pMOSFETs has been used to provide channel compressive strain to boost hole mobility [5]. However, the low solid solubility of Sn in Ge represents a challenge for the integration of

$\mathrm{Ge}_{1-\mathrm{x}} \mathrm{Sn}_{\mathrm{x}}$ technology due to the risk of precipitates or surface segregation [6].

In this work, doping of Ge with $\mathrm{Sn}$ atoms by ion implantation and solid phase epitaxial re-growth (SPER) is considered as a possible way to create thin $\mathrm{Ge}_{1-\mathrm{x}} \mathrm{Sn}_{\mathrm{x}}$ layers on Ge films deposited on $\mathrm{Si}$. In fact, the low diffusivity of $\mathrm{Sn}$ in Ge [7, 8] and the low temperature SPER [9] would enable the formation of thin $\mathrm{Ge}_{1-\mathrm{x}} \mathrm{Sn}_{\mathrm{x}}$ layers with limited thermal budget. However, ion implantation of high mass species on Ge is known to induce a characteristic "honeycomb" damage structure, impossible to anneal out with conventional thermal treatments [10, 11]. Ion implantation was thus carried out at liquid nitrogen temperature to avoid the void formation [12]. Three implant fluencies were tested: $5 \times 10^{15}, 1 \times 10^{15}$ and $5 \times 10^{14} \mathrm{at} / \mathrm{cm}^{2}$, respectively. Implant energy was set to $45 \mathrm{keV}$ and implants were carried out through an $11 \mathrm{~nm}$ nominally silicon nitride film $\left(114 \times 10^{15}\right.$ atoms $/ \mathrm{cm}^{2}$ at nominal stoichiometry) to prevent $\mathrm{Sn}$ out-diffusion. Samples were then annealed in inert atmosphere either at $350^{\circ} \mathrm{C}$ varying anneal time or for $100 \mathrm{~s}$ varying temperature from 300 to $500^{\circ} \mathrm{C}$. The results show that only the amorphous layers created by the two lowest fluence implants were annealed whereas the $5 \times 10^{15} \mathrm{at} / \mathrm{cm}^{2}$ implants left a $\sim 15 \mathrm{~nm}$ thick damaged layer even at $500^{\circ} \mathrm{C} 100 \mathrm{~s}$. In all cases, neither relevant $\mathrm{Sn}$ diffusion nor out-diffusion was detected and only a small relocation at the highest Sn concentration towards the SiN/Ge interface was observed. 


\section{EXPERIMENTAL}

Samples were obtained from 4 (100) Si wafers (4") where $1.5 \mu \mathrm{m}$ thick Ge films were epitaxially deposited by chemical vapor deposition (CVD). An $11 \mathrm{~nm}$ silicon nitride (SiN) cap was CVD deposited on top of the Ge films in order to inhibit Sn out-diffusion upon annealing. ${ }^{120} \mathrm{Sn}$ ions were implanted with energy of $45 \mathrm{keV}$ ( $7^{\circ}$ tilt). Three fluencies were implanted in 3 different wafers keeping them at liquid nitrogen temperature: $5 \times 10^{15}, 1 \times 10^{15}$ and $5 \times 10^{14} \mathrm{at} / \mathrm{cm}^{2}$, respectively. The fourth wafer was implanted with $5 \times 10^{15} \mathrm{at} / \mathrm{cm}^{2}$ fluence at room temperature as reference. $1 \times 1 \mathrm{~cm}^{2}$ samples were cut and thermally annealed by a rapid thermal process (RTP) system in $\mathrm{N}_{2}$ atmosphere. Temperature was varied between 300 and $500{ }^{\circ} \mathrm{C}$ whereas annealing time varied from 5 to $1200 \mathrm{~s}$.

Sample surface topography and possible nano-void formation was investigated by scanning electron microscopy (SEM, Jeol JMS 7401F) and atomic force microscopy (AFM, NT-MDT Solver P47). Amorphous layer (a-layer) thickness and damage recovering were measured by spectroscopic ellipsometry (SE) and channeling Rutherford backscattering spectrometry (RBS-c). SE measurements were performed using a variable angle ellipsometer (Horiba-Jobin-Yvon, UVISEL) at three different incident angles, i.e. $60^{\circ}, 65^{\circ}$ and $70^{\circ}$, respectively. The wavelength range was $280-800 \mathrm{~nm}$ with a step of $10 \mathrm{~nm}$. A four-phase model (air/ $\mathrm{Si}_{3} \mathrm{~N}_{4} / \mathrm{a}-\mathrm{Ge} / \mathrm{bulk} \mathrm{Ge}$ ) was used for the treatment of the data. In this configuration, it was considered that optical constants of the $\mathrm{Ge}_{1-\mathrm{x}} \mathrm{Sn}_{\mathrm{x}}$ a-layer can be modeled by a new amorphous dispersion formula. RBS-c was carried out at INFN - Laboratori Nazionali di Legnaro, Italy, using a $2 \mathrm{MeV}{ }^{4} \mathrm{He}^{+}$beam and two detectors with backscattering angles of $160^{\circ}$ and $120^{\circ}$ respectively and at the University of Surrey with $3.05 \mathrm{MeV}$ He with two detectors $\left(172^{\circ}, 148^{\circ}\right)$ specifically to look for $\mathrm{O}$ using the strong elastic resonance at $3038 \mathrm{keV}$. Sn depth distribution was determined by secondary ion mass spectrometry (SIMS) using a Cameca Wf/Sc-Ultra instrument. An $1 \mathrm{keV}\left(65^{\circ}\right) \mathrm{O}_{2}{ }^{+}$primary ion beam was used collecting positive secondary ions $\left({ }^{28} \mathrm{Si}^{14} \mathrm{~N}^{+},{ }^{74} \mathrm{Ge}^{+}\right.$and ${ }^{120} \mathrm{Sn}^{+}$) from a $66 \mu \mathrm{m}$ diameter area centered in a $200 \times 200$ $\mu \mathrm{m}^{2}$ rastered area. Sputtering time was converted to depth measuring the final crater depth with a mechanical profilometer. ${ }^{120} \mathrm{Sn}^{+}$intensity was converted to concentration with a relative sensitive factor (RSF) obtained by imposing the $5 \times 10^{14} \mathrm{at} / \mathrm{cm}^{2}$ nominal value to the measurement of the lowest fluence 'as implanted' sample.

\section{$\underline{\text { RESULTS }}$}

Figure 1 reports SEM images depicting the surface of the two samples implanted with $5 \times 10^{15}$ at $/ \mathrm{cm}^{2}$ fluence at room and liquid nitrogen temperature, respectively. It is evident that nano-voids formed under $\mathrm{Sn}^{+}$irradiation as expected from literature for high fluencies and high mass ion bombardment $[10,11]$. The voids are distributed on the sample surface with the typical 'honeycomb' symmetry. On the other hand, keeping the wafer at low temperature was successful in preventing the formation of such structures as shown in the left part of the figure. This indicates that the chosen ion implantation conditions formed only an a-layer and thus they are suitable to undergo a proper SPER process. AFM measurements of the surface roughness $(1 \times 1 \mu \mathrm{m} 2 \mathrm{scan}$ area $)$ showed identical root mean square values of $0.1 \mathrm{~nm}$ for bare wafers and samples implanted at liquid nitrogen temperature, and significantly higher values $(0.6 \mathrm{~nm})$ for the wafer implanted at room temperature. SE measured the following amorphous thickness values: $46.2,41.7$ and $35.6 \mathrm{~nm}$ for $5 \times 10^{15}, 1 \times 10^{15}$ and $5 \times 10^{14} \mathrm{at} / \mathrm{cm}^{2}$, respectively. RBS-c measured a $55 \mathrm{~nm}$ thick a-layer for the high fluence sample, i.e. a value compatible with SE data considering the limited depth resolution of RBS for those thicknesses.

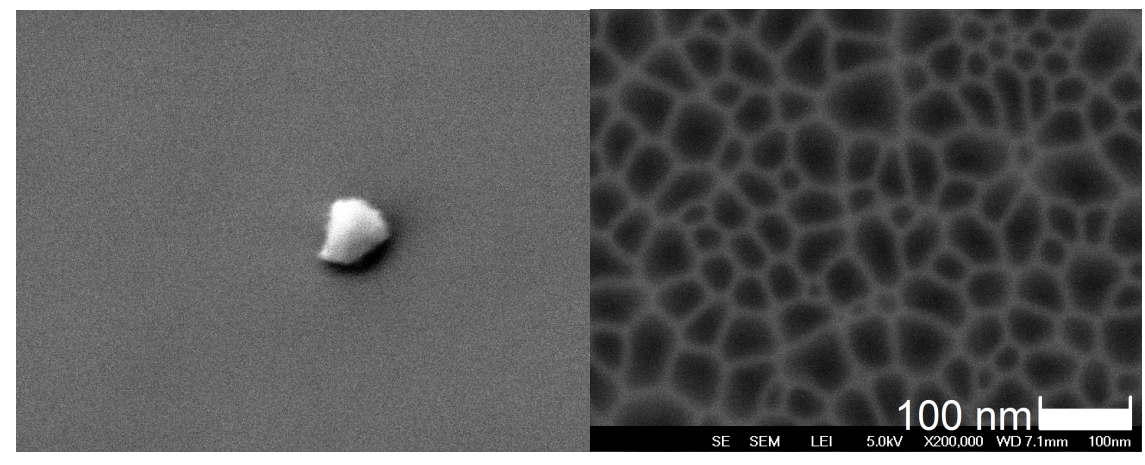


FIGURE 1. SEM plan view of the surface of the samples implanted with $45 \mathrm{keV}$ energy $\mathrm{Sn}^{+}$ions, $5 \times 10^{15}$ at $/ \mathrm{cm}^{2} \mathrm{fluence}$ at liquid nitrogen temperature (left) and room temperature (right). In the left picture a particle corroborates right focusing of the image.

Samples were thermally treated at $350^{\circ} \mathrm{C}$ varying annealing time from 5 to 1200 seconds. The SE measured alayer thicknesses are shown in Figure 2. Two behaviors depending on implanted fluence are observed: the $5 \times 10^{14}$ and $1 \times 10^{15} \mathrm{~cm}^{-2}$ implants are rapidly annealed out and the amorphous layer is fully re-crystallized after $60 \mathrm{~s}$ for both sample series. On the other side, the $5 \times 10^{15} \mathrm{at} / \mathrm{cm}^{2}$ series shows saturation in the re-growth of the a-layer and a "notcrystalline' phase of $\sim 15 \mathrm{~nm}$ seems to remain even after a $1200 \mathrm{~s}$ thermal treatment. RBS-c was carried out on the sample annealed at $350^{\circ} \mathrm{C}$ for $600 \mathrm{~s}$ : results confirmed that only $28 \mathrm{~nm}$ of the initial $55 \mathrm{~nm}$ a-layer was crystallized leaving a $27 \mathrm{~nm}$ amorphous thickness at the surface. Furthermore, in the remaining a-layer the same Sn fluence $\left(5.5 \times 10^{15} \mathrm{at} / \mathrm{cm}^{2}\right)$ measured on the 'as implanted' sample was detected. Figure 3 shows the a-layer thickness determined by SE for the samples isochronally treated for $100 \mathrm{~s}$. Once more, the $5 \times 10^{15} \mathrm{at} / \mathrm{cm}^{2}$ samples could not be completely annealed out even after a $500{ }^{\circ} \mathrm{C}$ treatment, differently from the medium and low fluence samples.

Tin depth distributions obtained by SIMS on samples annealed at $350{ }^{\circ} \mathrm{C}$ are shown in Figure 4. Curves are reported setting the zero point of the depth scale at the SiN/ Ge interface. The part of Sn profiles in SiN films is not properly quantified due to the lack of adequate standards. Furthermore, the Sn peak observed at the SiN/ Ge interface can fully or partially be a SIMS artifact due to changes of the ion yield. However, the comparison of the profiles is indicative of $\mathrm{Sn}$ distribution variations in $\mathrm{SiN}$ film and at the interface. The projected range of the 'as implanted' samples is at $\sim 10 \mathrm{~nm}$ depth ( $\mathrm{SiN}$ layer not included) for all the samples and the dopant is initially confined in the amorphous region. Amorphous/crystalline $(\mathrm{a} / \mathrm{c})$ interface determined by SE corresponds to $\sim 5 \times 10^{19}$ $\mathrm{at} / \mathrm{cm}^{3}$ concentration for all the 3 samples. Measured fluences are close to nominal values and are reported in the legend of the figure. Only the high fluence implant turned out with an appreciable lower value, i.e. $4.1 \times 10^{15}$ against $5 \times 10^{15} \mathrm{at} / \mathrm{cm}^{2}$. However, this sample shows also higher ${ }^{74} \mathrm{Ge}^{+}$intensity at depth $0-15 \mathrm{~nm}$ with respect to the equilibrium value observed in the deeper part of the profiles. This 'bump' corresponds to the same high $\mathrm{Sn}$ concentration layer that is not possible to anneal according to SE and RBS. Therefore a possible alteration of ion yield affecting the quantification approach in this region can be expected.

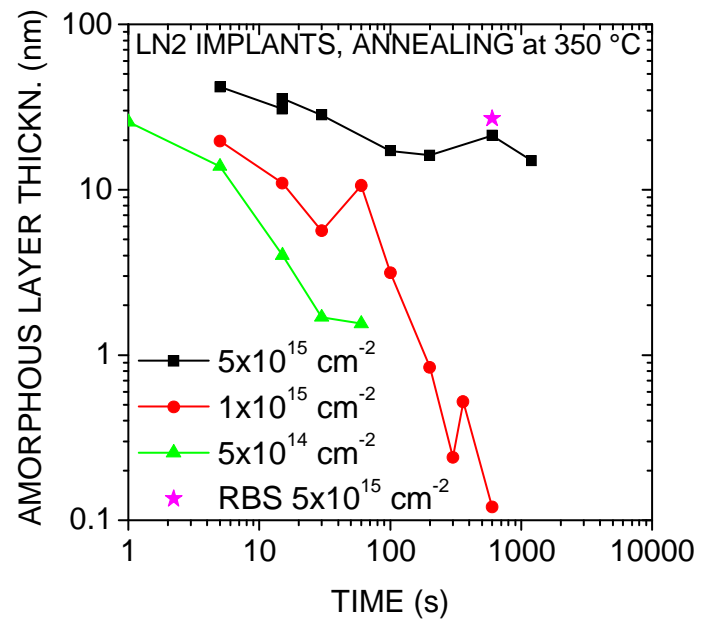

FIGURE 2. Amorphous layer thickness measured by spectroscopic ellipsometry on the three fluence series samples annealed at $350{ }^{\circ} \mathrm{C}$ for different times. The star symbol indicates the values obtained by RBS-c. Initial a-layer thicknesses were $46.2(55 \mathrm{~nm}$ for RBS), 41.7 and $35.6 \mathrm{~nm}$ for $5 \times 10^{15}, 1 \times 10^{15}$ and $5 \times 10^{14} \mathrm{at} / \mathrm{cm}^{2}$, respectively.

Thermal treatments did not induce any relevant Sn diffusion in any sample. The same no-diffusion is observed for the isochronal treatments for 100s (data not shown) confirming a low diffusivity for Sn in Ge. Furthermore, measured Sn fluencies are constant within SIMS accuracy upon annealing for the 3 series of samples indicating that SiN cap prevented Sn out-diffusion. Varying amounts of oxygen were measured in this sample set, up to $30 \times 10^{15}$ $\mathrm{O} / \mathrm{cm}^{2}$.

SIMS profiles reported in linear scale revealed some $\mathrm{Sn}$ relocation after SPER process as depicted in Figure 5. The $\mathrm{a} / \mathrm{c}$ interface positions as determined by SE are reported as vertical dashed lines in the plots: it is evident that re-growth of the a-layer is slowed down by high Sn concentrations. After a $30 \mathrm{~s}$ annealing the Sn peak observed at the $\mathrm{SiN} / \mathrm{Ge}$ interface increases, suggesting a small segregation of tin when the a/c interface reaches the high 
concentration part of the profiles. Finally, in the high dose samples, the $\mathrm{Sn}$ distributions have a shoulder at $\sim 18 \mathrm{~nm}$, i.e. very close to the $\mathrm{a} / \mathrm{c}$ interface according to SE. This is considered an indication of possible precipitates or different phases occurring at high Sn concentration.

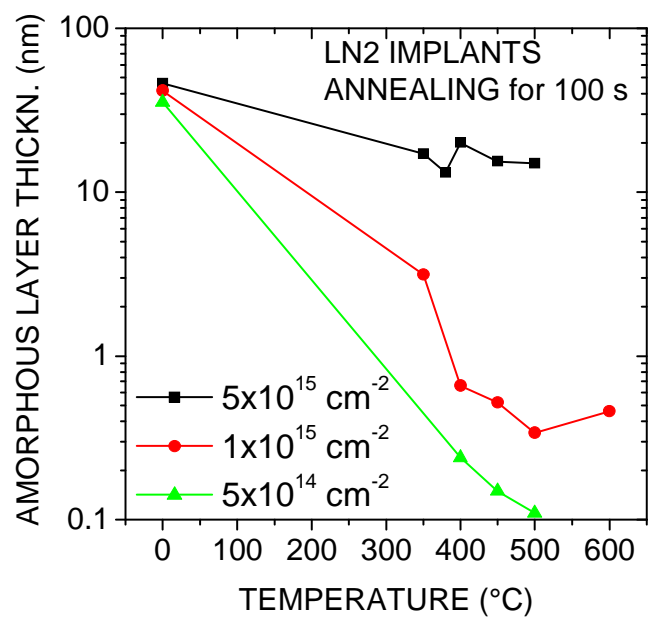

FIGURE 3. Amorphous layer thickness measured by spectroscopic ellipsometry on the three sample series each annealed for $100 \mathrm{~s}$ with varying temperature.

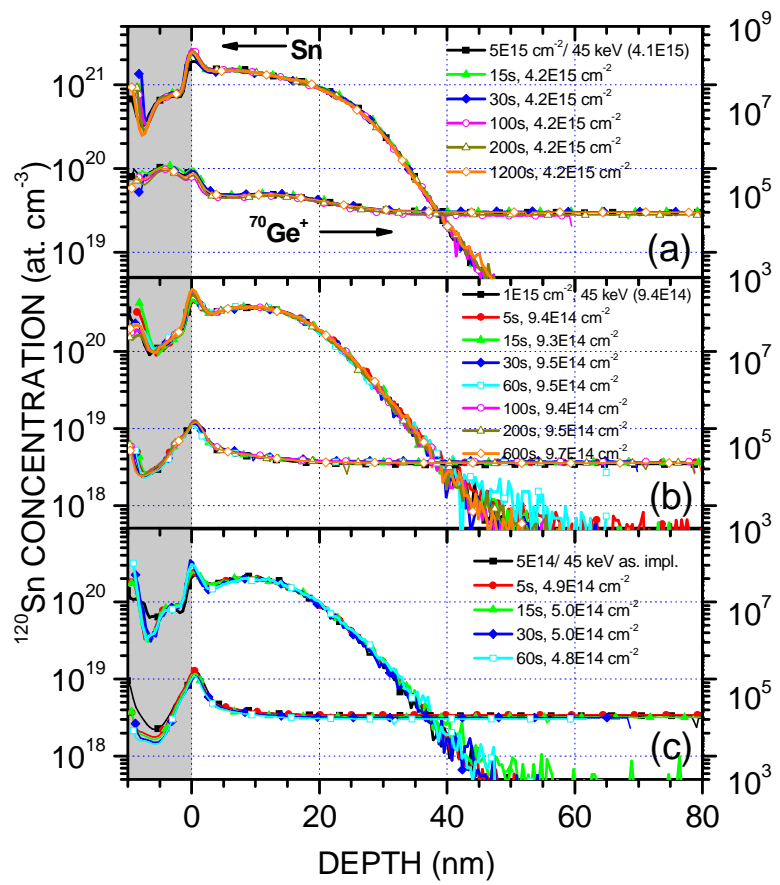

FIGURE 4. SIMS profiles obtained on $5 \times 10^{15}$ at $/ \mathrm{cm}^{2}$ (a), $1 \times 10^{15}$ (b) and $5 \times 10^{14}$ (c). ${ }^{120} \mathrm{Sn}$ quantified profiles refer to the left axis whereas qualitative ${ }^{74} \mathrm{Ge}^{+}$intensities are related to right axis. The origin of the depth scale was set at the SiN/Ge interface. 


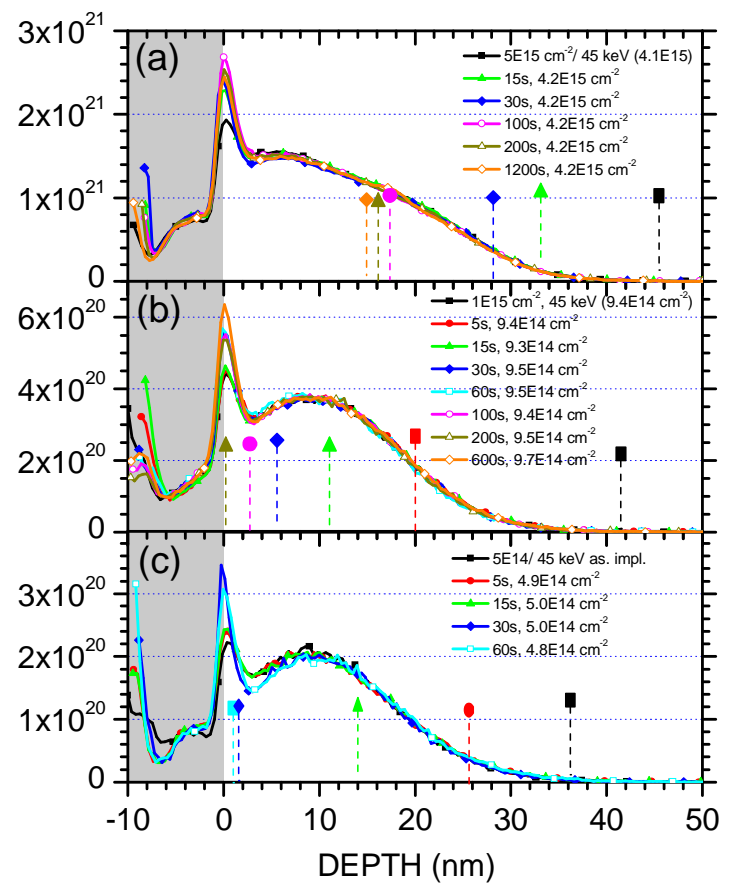

FIGURE 5. SIMS profiles obtained on $5 \times 10^{15}$ at $/ \mathrm{cm}^{2}$ (a), $1 \times 10^{15}$ (b) and $5 \times 10^{14}$ (c). Vertical dashed lines identify the a/c interface as measured by SE.

\section{DISCUSSION AND CONCLUSION}

Solid phase epitaxial re-growth was studied on tin implanted thin films $(1.5 \mu \mathrm{m})$ of epitaxial Ge implanted at liquid nitrogen temperature by high fluence ${ }^{120} \mathrm{Sn}^{+}$ions (implant energy $45 \mathrm{keV}$, fluence range of $5 \times 10^{14}-5 \times 10^{15}$ $\mathrm{at} / \mathrm{cm}^{2}$ ) through a nominally $11 \mathrm{~nm} \mathrm{SiN} \mathrm{cap,} \mathrm{deposited} \mathrm{to} \mathrm{prevent} \mathrm{out-diffusion.} \mathrm{High} \mathrm{fluence} \mathrm{was} \mathrm{chosen} \mathrm{in} \mathrm{order} \mathrm{to}$ form

thin

$\mathrm{Ge}_{1-\mathrm{x}} \mathrm{Sn}_{\mathrm{x}}$ layers with $\mathrm{Sn}$ concentrations in the range of $0.5-4 \%$. Low temperature implantation was effective to prevent the characteristic formation of nano-voids ('honeycombing') in Germanium when irradiated at high fluences $\left(>5 \times 10^{14} \mathrm{at} / \mathrm{cm}^{2}\right)$ with high mass ions. Amorphous layers were formed with $36-46 \mathrm{~nm}$ thickness depending on fluence as expected. SPER was carried out in $\mathrm{N}_{2}$ atmosphere at either $350^{\circ} \mathrm{C}$ temperature varying annealing time or varying temperature from 300 to $500^{\circ} \mathrm{C}$ for $100 \mathrm{~s}$. The $350^{\circ} \mathrm{C}$ treatment showed a SPER rate depending on Sn concentration, being slower the higher the Sn concentration. However, the $5 \times 10^{15} \mathrm{at} / \mathrm{cm}^{2}$ samples remained with a residual 'non-crystalline' Ge layer of $15-20 \mathrm{~nm}$ corresponding to the high $\mathrm{Sn}$ concentration region of the depth profile. SIMS profiles showing a shoulder at $18 \mathrm{~nm}$ suggests the possible formation of precipitates of a new phase or Sn immobile clusters. No relevant diffusion of Sn was detected for the annealing processes investigated and only a small segregation at the $\mathrm{SiN} / \mathrm{Ge}$ interface was observed after a $350^{\circ} \mathrm{C} 100$ s annealing or higher thermal budgets.

Conventional annealing faces challenges to recover relatively high Sn-doped Ge a-layers. Therefore new 'nonequilibrium' annealing schemes (laser annealing, FLASH annealing,...) should be considered in order to overcome the problems related to Sn precipitation upon SPER.

\section{ACKNOWLEDGMENTS}

This work has been supported by the European Community as an Integrating Activity 'Support of Public and Industrial Research Using Ion Beam Technology (SPIRIT)' under EC contract no. 227012. Prof. G. Ottaviani and Dr. R. Tonini of University of Modena and Reggio Emilia are greatly acknowledged for RBS measurements. FM's 
research is carried out as part of the DART project ("Dopant profile and activation research for advanced CMOS technology"), supported by the Provincia Autonoma di Trento and the European Union within the Marie Curie COFUND program.

\section{REFERENCES}

1. G. He and H.A. Atwater, Phys. Rev. Lett. 79, 1937 (2007).

2. J.D. Sau and M.L. Cohen, Phys. Rev. B 75, 045208 (2007)

3. S. Gupta et al., "GeSn technology: Extending the Ge electronics roadmap" in IEEE International Electron Devices Meeting (IEDM) - 2011, Institute of Electrical and Electronics Engineers (IEEE) Proceedings, pp. 16.6.1-16.6.4.

4. G. Han et al., "High-mobility germanium-tin (GeSn) P-channel MOSFETs featuring metallic source/drain and sub-370 ${ }^{\circ} \mathrm{C}$ process modules" in IEEE International Electron Devices Meeting (IEDM) - 2011, Institute of Electrical and Electronics Engineers (IEEE) Proceedings, pp. 16.7.1-16.7.3

5. B. Vincent et al., Microelectronic Eng. 88(4), 342, (2011).

6. J. P. Fleurial and A. Borshchevsky, J. Eletrochem. Soc. 137(9), 2928 - 2937 (1990).

7. P. Kringhofj and R. G. Elliman, Appl. Phys. Lett. 65(3), 324 (1994).

8. M. Friesel, U. Södervall, W. Gust, J. Appl. Phys. 78(9), 5351 (1995).

9. A. Claverie et al., Thin Solid Films 518, 2307 (2010).

10. I.H. Wilson, J. Appl. Phys. 53(3), 1698 (1982).

11. L. Romano et al., J. Appl. Phys. 107, 084314 (2010).

12. G. Impellizzeri et al, J. Appl. Phys. 106, 013518 (2009). 